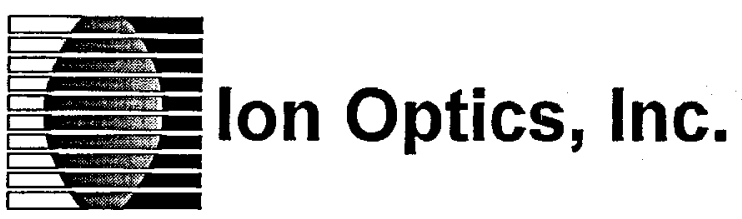

April, 2001

\author{
Final Report for
}

\title{
Frequency Selective Surfaces for Rugged Thermophotovoltaic Emitters
}

DOE grant: DE-FG02-00ER83026

Project Period: $\quad$ September 29, 2000-March 01, 2001

Report Date: $\quad$ April 2001

Prepared by:

Ion Optics, Inc.

411 Waverly Oaks Road

Suite 144

Waltham, MA 02452

POC: Jim Daly, (781) 788-8777 x 118, jdaly@ion-optics.com

Submitted to:

SBIR Program Manager

SC-32 RooomE-209

U.S. Department of Energy

19901 Germantown road

Germantown, MD 20874-1290

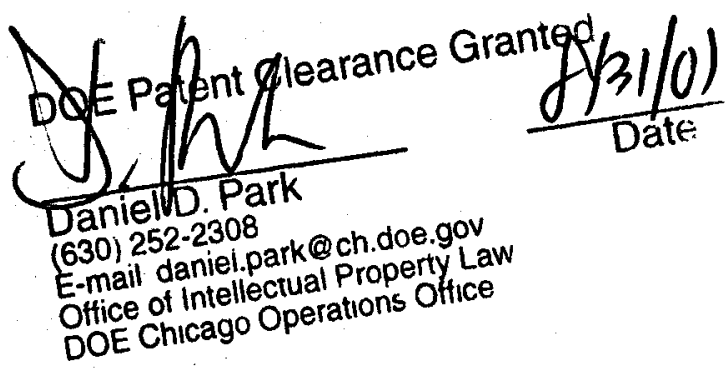




\section{DISCLAIMER}

This report was prepared as an account of work sponsored by an agency of the United States Government. Neither the United States Goverament nor any agency thereof, nor any of their employees, makes any warranty, express or implied, or assumes any legal liability or responsibility for the accuracy, completeness, or usefulness of any information, apparatus, product, or process disclosed, or represents that its use would not infringe privately owned rights. Reference herein to any specific commercial product, process, or service by trade name, trademark, manufacturer, or otherwise does not necessarily constitute or imply its endorsement, recommendation, or favoring by the United States Governmeat or any ageacy thereof. The views and opinions of authors expressed herein do not necessarily state or reflect those of the United States Government or any agency thereof. 


\section{DISCLAIMER}

Portions of this document may be illegible in electronic image products. Images are produced from the best available original document. 


\section{SUMMARY}

Thermophotovoltaic (TPV) power generation is an alternate physical process to thermoelectric power generation. TPV uses photovoltaic cells to convert near infrared radiation from a hot body to electricity. Process efficiency can be increased if the surface of the hot body is covered with selective emitters. An expensive alternate to use of selective emitters is the placement of reflective filters over the photovoltaic cell.

Current TPV selective emitters are fabricated from rare earth oxides. They are fragile and do not survive many cycles of heating and cooling to high temperatures. In addition, they emit only about $40 \%$ of available energy in the selected band. Ion Optics developed a surface texture that acts as a selective emitter using principles of photonic bandgap crystals. This texture was replicated in gold on silicon and platinum on aluminum oxide surfaces. Total radiated energy increased with increasing temperature, but the relative magnitude of emission as a function of wavelength did not change. In-band power exceeded $40 \%$ of the total.

Experiments showed control of the wavelength of this emission by altering surface geometry, and that the fine geometry can be duplicated in materials systems capable of operating for extended periods of time at high temperatures in air. 


\section{OBJECTIVES}

The feasibility of using micro-patterned textured surfaces as selective emitters for TPV was demonstrated in this phase 1 SBIR research program. All technical objectives were achieved.

Phase 1 technical objectives, directly copied from the original proposal, were:

1. Model surface emission as a function of geometry and predict an "optimum" configuration for initial tests.

2. Design the emitter, including choice of metals, choice of fabrication technique, and lithographic patterns.

3. Fabricate the emitter

4. Measure emitter optical properties at low temperature

5. Measure emitter optical properties at high temperature

6. Ascertain suitability to long term atmospheric exposure and a large number of thermal cycles.

Limitations of time and funding for phase 1 prevented any attempt, as noted in the original proposal, at inclusion of the new selective emitter in existing TPV systems. Due to fabrication problems, later resolved, the surface with the smallest features that emitted at the wavelength closest to desired values for thermophotovoltaics (TPV) could not be heated to the highest temperatures.

\section{THEORETICAL MODEL OF SURFACE EMISSION}

Narrow bandwidth emission from a textured surface requires that the surface act as a "photonic crystal". The electromagnetic energy in light interacts with the surface structure and average properties of the substrate including electrical conductivity, index of refraction and dielectric constant. The light does not interact with the silicon crystal (atomic structure). Optimization of surface structure to meet the demands requires a complex calculation.

Prof. Rana Biswas of Iowa State University performed the calculations for this work. All calculations and data in this section were funded by a NIST contract. ${ }^{1}$ Data is being reproduced here as background material to show how our theoretical and experimental data agree in determination of scaling rules for predicting emission spectra from photonic bandgap materials. A large, multi-processor computer system at AMES Laboratory, IA was utilized for digital calculations. ${ }^{2}$ The transfer matrix method was chosen to match desired input parameters.

Ion Optics emitter can be described as a two-dimensional photonic bandgap (PBG) structure. Periodic surface structure is imposed upon a thick silicon substrate. There are possible metal coatings, also patterned, on top of this structure. Metal coatings are thin and of the same pattern as the etched pits in silicon. Therefore, there is really only one layer of active photonic material. The theoretical problem is to calculate the absorption as a function of wavelength for different etched patterns. We are assuming that emission would follow the absorption curve by Kirchoff's Law. The goal of these simulations was to find the optimum pattern that gives narrow, strong absorption peaks. Preferably just one peak, or if many peaks then they should be well separated. 
Calculations were performed for etched pits in the shape of a cross, square, and round hole. The "pit" was modeled on a finite element grid and the pattern was assumed to repeat itself endlessly over the surface to give boundary conditions. Calculation of transmission and reflection from this structure could be interpolated to give absorption, so that the transfer matrix method seemed to be as optimum approach.

The transfer matrix relates the incident fields on one side of the photonic crystal with the outgoing fields on the other side. This method calculates the transmission and reflection properties of electromagnetic (EM) waves of various frequencies incident on a finite thickness slab of PBG material.

Let $a, b, c$ be the grid spacing of the finite element array in the $x, y, z$ directions. Fields are known in the $x-y$ plane at $z=0$ (front and back surfaces). Then Maxwell's equations:

$$
\Delta \times E=i\left(\omega / c^{\prime}\right) H, \Delta \times H=-i\left(\omega / c^{\prime}\right) D
$$

are integrated to find the fields at $\mathrm{z}=\mathrm{c}$. Here, we have used c' as the speed of light to distinguish it from the unit cell distance $c$ in the $z$ direction. The specific equations to find the fields on the next plane (at point $r=c$ ) in terms of the fields on the previous plane are:

$$
\begin{aligned}
& \mathrm{E}_{\mathrm{x}}(\mathbf{r}+\mathbf{c})=\mathrm{E}_{\mathrm{x}}(\mathbf{r})+\mathrm{ic} \omega \mu_{\mathrm{o}} \mu(\mathbf{r}) \mathrm{H}_{\mathrm{y}}(\mathbf{r}) \\
& +\left(\mathrm{ic} / \mathrm{a} \omega \varepsilon_{0} \varepsilon(\mathbf{r})\right)\left[\mathbf{a}^{-1}\left\{\mathrm{H}_{\mathrm{y}}(\mathbf{r}-\mathbf{a})-\mathrm{H}_{\mathrm{y}}(\mathbf{r})\right\}-\mathbf{b}^{-1}\left\{\mathrm{H}_{\mathrm{x}}(\mathbf{r}-\mathbf{b})-\mathrm{H}_{\mathrm{x}}(\mathbf{r})\right\}\right] \\
& +\left(\mathrm{ic} / \mathbf{a} \omega \varepsilon_{0} \varepsilon(\mathbf{r}+\mathbf{a})\right)\left[\mathbf{a}^{-1}\left\{\mathrm{H}_{\mathbf{y}}(\mathbf{r})-\mathrm{H}_{\mathbf{y}}(\mathbf{r}+\mathbf{a})\right\}-\mathbf{b}^{-1}\left\{\mathrm{H}_{\mathrm{x}}(\mathbf{r}+\mathbf{a}-\mathbf{b})-\mathrm{H}_{\mathrm{x}}(\mathbf{r}+\mathbf{a})\right\}\right] \\
& E_{y}(r+c)=E_{y}(r)-i c \omega \mu_{0} \mu(r) H_{x}(r) \\
& +\left(\mathbf{i c} / \mathbf{b} \omega \varepsilon_{0} \varepsilon(\mathbf{r})\right)\left[\mathbf{a}^{-1}\left\{\mathrm{H}_{\mathbf{y}}(\mathbf{r}-\mathbf{a})-\mathrm{H}_{\mathbf{y}}(\mathbf{r})\right\}-\mathbf{b}^{-1}\left\{\mathrm{H}_{\mathbf{x}}(\mathbf{r}-\mathbf{b})-\mathrm{H}_{\mathbf{x}}(\mathbf{r})\right\}\right] \\
& +\left(\mathrm{ic} / \mathrm{b} \omega \varepsilon_{0} \varepsilon(\mathbf{r}+\mathbf{b})\right)\left[\mathbf{a}^{-1}\left\{\mathrm{H}_{\mathbf{y}}(\mathbf{r}-\mathbf{a}+\mathbf{b})-\mathrm{H}_{\mathbf{y}}(\mathbf{r}+\mathbf{b})\right\}-\mathbf{b}^{-1}\left\{\mathrm{H}_{\mathbf{x}}(\mathbf{r})-\mathrm{H}_{\mathbf{x}}(\mathbf{r}+\mathbf{b})\right\}\right] \\
& H_{x}(r+c)=H_{x}(r)+i c \omega \varepsilon_{0} \varepsilon(r+c) E_{y}(r+c) \\
& +\left(i c / a \omega \mu_{0} \mu(r-a+c)\right)\left[a^{-1}\left\{E_{y}(r+c)-E_{y}(r-a+c)\right\}-b^{-1}\left\{E_{x}(r-a+b+c)-E_{x}(r-a+c)\right\}\right] \\
& +\left(i c / a \omega \mu_{0} \varepsilon(r+c)\right)\left[a^{-1}\left\{E_{y}(r+a+c)-E_{y}(r+c)\right\}-b^{-1}\left\{E_{x}(r+b+c)-E_{x}(r+c)\right\}\right] \\
& H_{y}(r+c)=H_{y}(r)+i c \omega \varepsilon_{0} \varepsilon(r+c) E_{x}(r+c) \\
& +\left(i c / a \omega \mu_{0} \mu(r-b+c)\right)\left[a^{-1}\left\{E_{y}(r+a+b+c)-E_{y}(r-b+c)\right\}-b^{-1}\left\{E_{x}(r+c)-E_{x}(r-b+c)\right\}\right] \\
& +\left(i c / a \omega \mu_{0} \varepsilon(r+c)\right)\left[a^{-1}\left\{E_{y}(r+a+c)-E_{y}(r+c)\right\}-b^{-1}\left\{E_{x}(r+b+c)-E_{x}(r+c)\right\}\right]
\end{aligned}
$$

Iowa State University (ISU) simulated the reflection, transmission and absorption of the 3-dimensional multilayer stack of the etched silicon surface with transfer matrix simulations on parallel computing systems. ISU found that the absorption of the silicon wafer was significant because of the large thickness. Initial calculations were also unstable and unduly influenced by coherent back-surface reflections. Back reflections were suppressed by gradually changing the 
index of refraction of silicon from 3.4 to a value of 1 in 36 layers. [In the real world, the back surface of the silicon wafer is bright etched and reflections are scattered.] The change stabilized the numerical results, with reduced statistical fluctuations. However, agreement with experimental data was only fair. The next change was to add the effect of surface plasmons, per work of Ebbesen et al. $3,4,5,6,7,8$

On polished, smooth surfaces light will not couple into the surface plasmons because the wavevector satisfies this relationship (Figure 1):

$$
k_{x}^{i n c}=\frac{\omega}{c} \sin \theta<\frac{\omega}{c}
$$

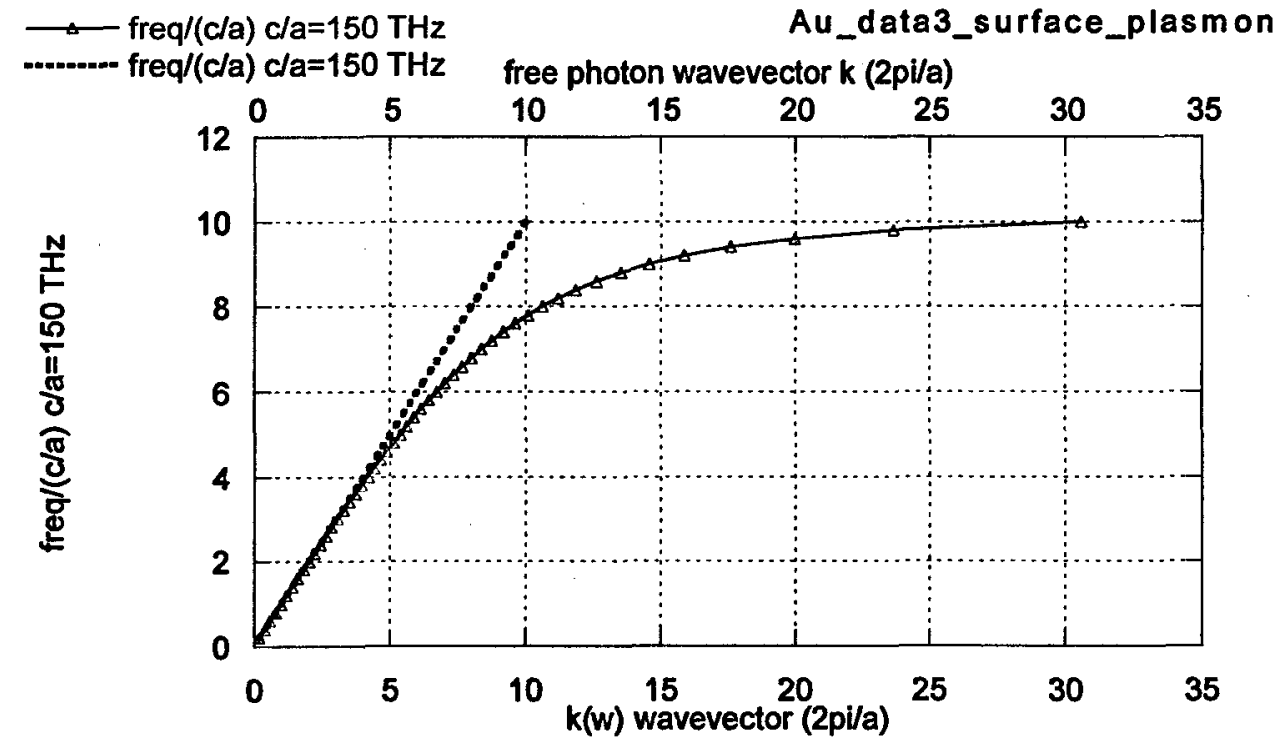

Figure 1 Surface plasmon frequency for gold.

Using a periodic pattern induces wavectors $\mathbf{G}$ on the surface according to the equations below. Surface states induced by the periodic geometry allow photons to couple to surface plasmons. There are strong resonance peaks at wavelengths proportional to the geometry of the structure (figure 2).

$$
\begin{gathered}
\frac{\omega}{c} \sin \theta \mathbf{i}+\mathbf{G}=\mathbf{k}_{s p} \\
\frac{\omega}{c} \sin \theta(\cos \phi \mathbf{x}+\sin \phi \mathbf{y}) \pm i G \mathbf{x} \pm j G \mathbf{y}=k_{s p}=\frac{\omega}{c}\left(\frac{\varepsilon 1 \varepsilon 2}{\varepsilon 1+\varepsilon 2}\right)^{1 / 2} \mathbf{u} \\
\left(\frac{\omega}{c}\right)^{2}\left(\frac{\varepsilon 1 \varepsilon 2}{\varepsilon 1+\varepsilon 2}\right)=\left(\frac{\omega}{c}\right)^{2}\left[\left(\sin \theta \cos \phi \pm \frac{i G_{x}}{\omega / c}\right)^{2}+\left(\sin \theta \sin \phi \pm \frac{j G_{y}}{\omega / c}\right)^{2}\right]
\end{gathered}
$$


$k(w)$ Surf Plasm

$k(w)$ free photon (FP) $\quad$ surface plasmon dispersion; square lattice $a=2 \mu$

$k(w)$ FP 10 deg

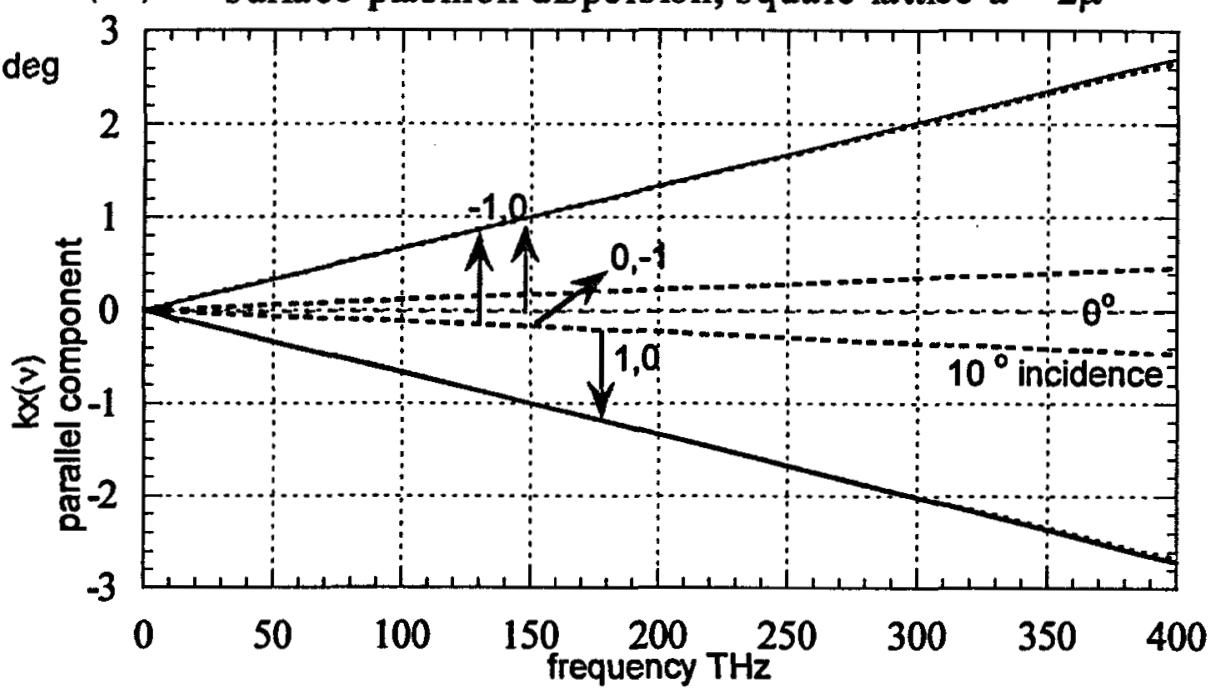

Figure 2 Surface plasmon dispersion for square lattice

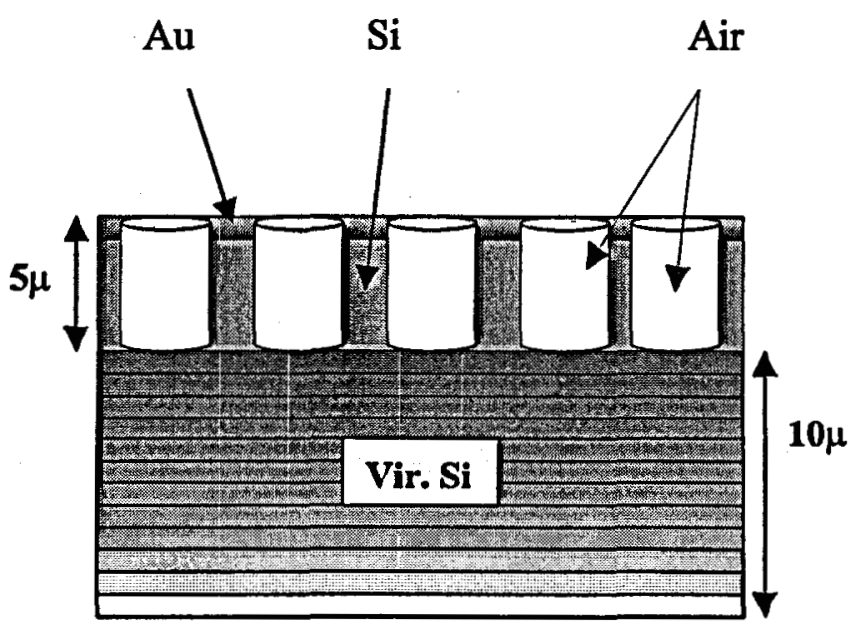

Figure 3, Geometry of calculation for photonic bandgap model. The top gold layer has a minimum thickness of one lattice unit which is one micron (actual thickness is about 0.1 micron). The substrate thickness is simulated by a gradual change in dielectric constant that inhibits reflections from the back surface and improves stability of the numerical calculation.

The model shown in figure 3 was used to calculate the absorption (emission) peaks (figure 4, right) of Ion Optics samples. These results compare well with measured data (figure 4, left) where the short wavelength cutoff of the instrument deleted one peak. 

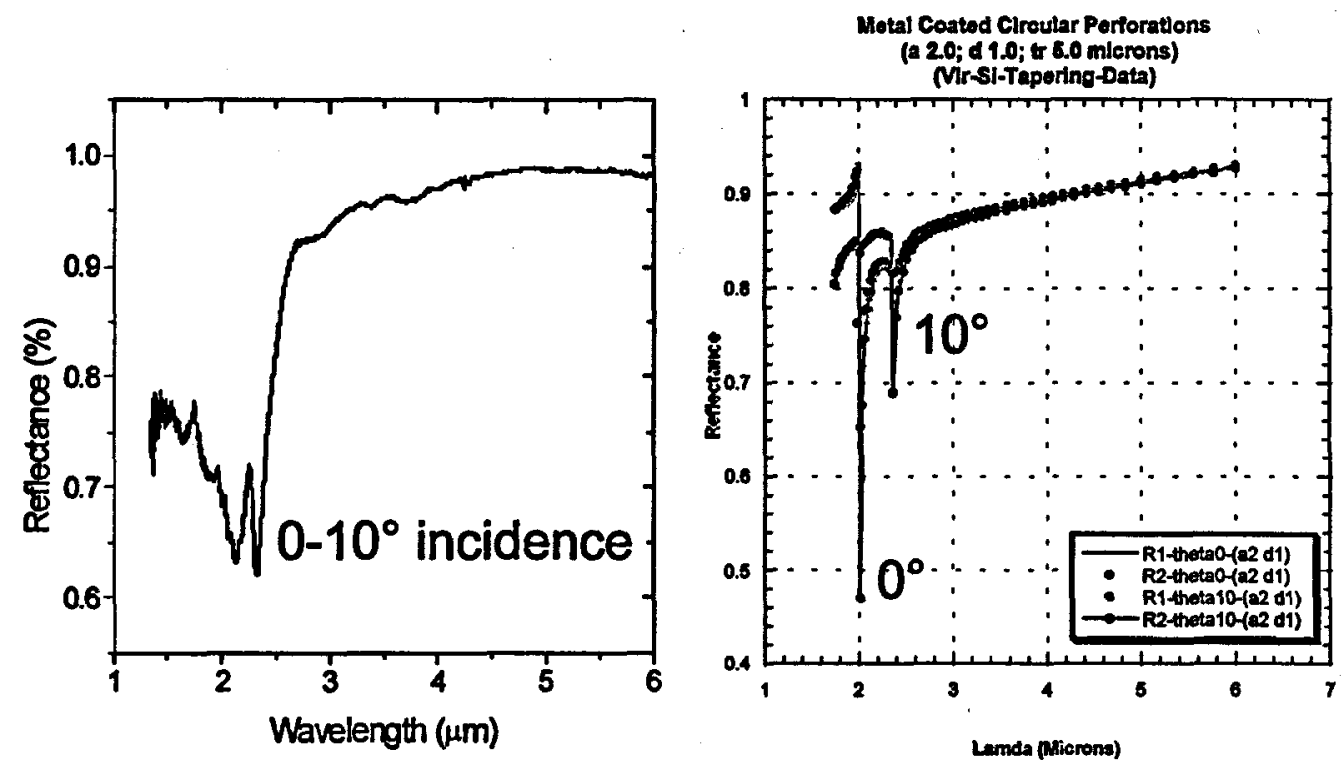

Figure 4 Experimental reflectance data collected from a silicon photonic crystal of round holes on a 2-micron square lattice, $350^{\circ} \mathrm{C}$ (left). The collection angle was from 0$10^{\circ}$. The first principles computational solution for the same photonic lattice calculated at $0^{\circ}$ and $10^{\circ}$ incidence. (Right)

\section{DESIGN THE EMITTER, INCLUDING CHOICE OF METALS, CHOICE OF FABRICATION TECHNIQUE, AND LITHOGRAPHIC PATTERNS.}

At the time the phase 1 proposal was written, the suggested surface structure to obtain the desired emittance was a "thick" layer of metal, a few microns deep, with fine structures extending from top to bottom. We proposed to fabricate this structure from super alloys such as $\mathrm{Ni}-\mathrm{Cr}$-Co-Al-Y used as bond layers and oxygen barriers for the high temperature turbine engine blades. However, a few months later when work on phase 1 started, new data suggested that the preferred structure was a very thin film of metal on the surface of a non-metallic substrate which could be air (unsupported foil) or an insulator or a semiconductor. Very thin films could not be obtained for the super alloy that is deposited in production by plasma spray with a "rough" surface, unsuitable for $150 \mathrm{~nm}$ thick films.

For our phase 1 experiments with highly polished very thin films, we wanted to work with materials that could be easily processed. Because the proposed application would be limited to $900^{\circ} \mathrm{C}$, because silicon is very cheap and easy to process with existing microelectronic fabrication lines, and because gold has very high infrared reflectance, we initially used gold on silicon for proof-of-principle experiments. Later, for high temperature testing, we switched to platinum on $\mathrm{Al}_{2} \mathrm{O}_{3}$.

Experiments on samples tested at low temperatures were partially funded by other programs. Experiments on samples tested at high temperatures was funded only by the present phase 1. Initially, standard photolithography was used to create the patterns needed. This was a 
less expensive option when we wanted to try out a large number of different patterns, varying the pattern size, spacing, geometrical arrangement, and geometry of the etched holes (figure 1). Because we were initially concerned about the adhesion of the thin metal film to the substrate when exposed to many thermal cycles, the gold layer was deposited directly on top of the silicon wafer with a metallic adhesion/diffusion barrier layer. Unfortunately, as the temperatures were increased to the region of interest to this program, this metallic structure interacted (the diffusion barrier failed) and a gold-silicon intermetallic phase was formed. Visible optical appearance of the surface changed from yellow gold, to a diffuse white finish. He infrared properties of the surface were altered to a lesser extent (figure 5), but sample structure was changed.

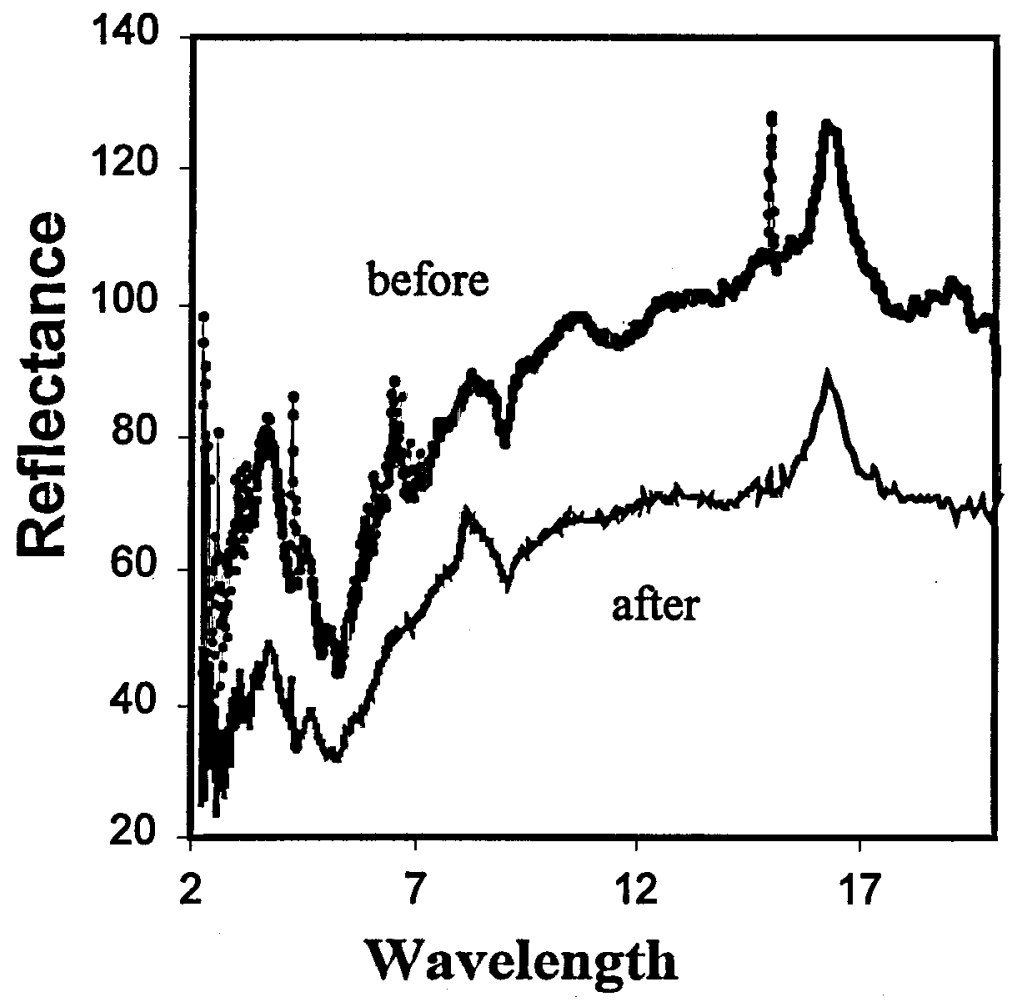

Figure 5 Reflectance (percent) as a function of wavelength (microns) of gold with metallic bonding layer before and after heating to $500^{\circ} \mathrm{C}$ in air. Emissivity peak is minimum in absorption at about 6 microns.

Samples for this program were then fabricated of gold on ceramic bonding-diffusion barrier layer on silicon for modest temperature testing, and on polished aluminum-oxide substrates for high temperature testing.

\section{FABRICATE THE EMITTER}

Some of this work was performed for other programs. Only those samples specifically designated for testing at high temperature were charged to the phase 1 effort.

Theoretical work discussed in section 1 implied that a very thin layer of metal on a dielectric substrate would give optimum results. To obtain a thin, high quality surface film, one must start with a polished substrate or the surface film would be discontinuous. For experiments, 
it was faster and less expensive to obtain polished silicon and sapphire wafers for low and high temperature tests respectively. These wafers were cleaned, then coated with metal by evaporation. For silicon substrates, the metallization was either $5 \mathrm{~nm}$ Ti followed by about $150 \mathrm{~nm}$ of gold, or the surface was first coated with about $100 \mathrm{~nm}$ of silicon-nitride followed by an identical metallization. Titanium was believed needed as an adhesion layer. Gold was chosen because it has the highest possible electrical conductivity, and infrared reflectivity.

Gold films on silicon wafers were patterned by photolithography using standard masks produced by electron beam writing. A dense array of features such as is used by our designs takes a great deal of "write" time on systems used to create masks. While simple masks used for electronic devices can cost about $\$ 1,000$ each for the size desired, vendors quoted upwards of $\$ 100,000$ for our work on a fully dense masks for $100 \mathrm{~mm}$ wafers. (They did not want the work!) The Jet Propulsion Laboratory produced some masks (figure 6) writing only a small fraction of the total area.

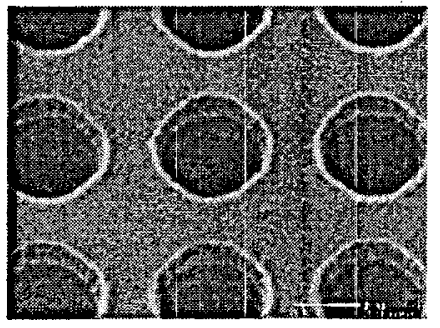

17A-006

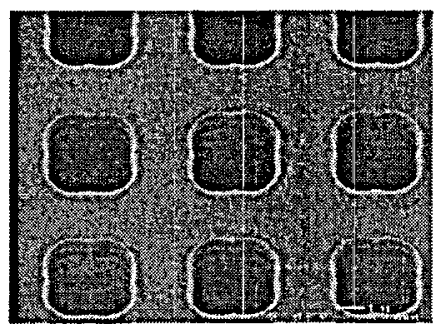

17A-004

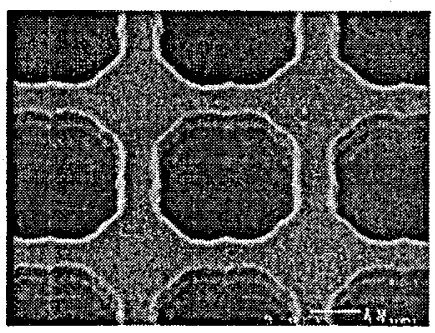

17A-001

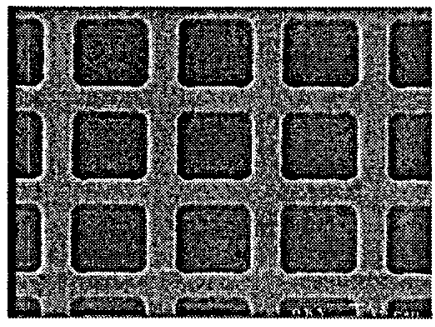

17A-002

Figure 6 Scanning electron micro-photographs of initial selective emitter surfaces. Dimensions in microns giving hole length and center to center spacing: top left 1x1.5, top right $2 \times 3$, bottom left $2 x 4$, bottom right $4 x 6$.

Patterns from the mask set were transferred to the metallized wafers by photolithography and deep reactive ion etching (RIE). The metal coated substrate was coated with photoresist, pre-treated, exposed to uv light through the patterned mask, and developed. The patterned resist coated wafer was then etched in vacuum by RIE, and then residual photoresist was removed. Typical etch depth was 5 microns, going through the metal layer and deep into the substrate. Results are shown in figure 6 above. Note that all patterns on the masks were square, but that the smallest pattern was not clearly resolved by the existing optical system and "corner rounding" led to a circular pattern. Test results are discussed in the next section. 
All work with sapphire was directly connected to this TPV program. Small diameter sapphire wafers used were coated with $150 \mathrm{~nm}$ of platinum. Some substrates were coated directly without an adhesion layer, some samples were first coated with $5 \mathrm{~nm}$ of titanium for adhesion, as before. Without the adhesion layer, samples were annealed at $700^{\circ} \mathrm{C}$ for 2 hours to remove any thermal strain in the metal film, promoting better adhesion. To create a pattern of smaller features, as the then current photo-processing laboratory did not seem capable, Ion Optics turned to holographic lithography. The work was performed by the nano-fabrication laboratory at Massachusetts Institute of Technology. Briefly, the diffraction pattern of a laser beam from a single aperture, by reflection from mirrors at 90 -degree angles, can be made to reproduce itself uniformly over a large area. The resulting pattern of round holes in a square area exactly matches the pattern desired for IOI emitters.

Metal-coated sapphire wafers were coated with a polymer antireflection layer, then a thin layer of silicon dioxide, and then a layer of photoresist. The AR coating is needed to prevent reflection from the polished metal surface altering the calibrated photo-exposure of resist. The layer of silica is needed to transfer the pattern from the exposed resist, through the AR coating. The top layer of photoresist is actually changed, chemically, by exposure to the diffraction pattern of laser light. When patterning is complete, a layer of AR coating, silica and photoresist is left on the surface to act as a mask for etching. The metal was etched by ion bombardment and then the remaining photoresist was removed. Figure 7 shows the test pattern during processing. The pattern is nominally 0.5 -micron diameter holes spaced 1 micron apart.

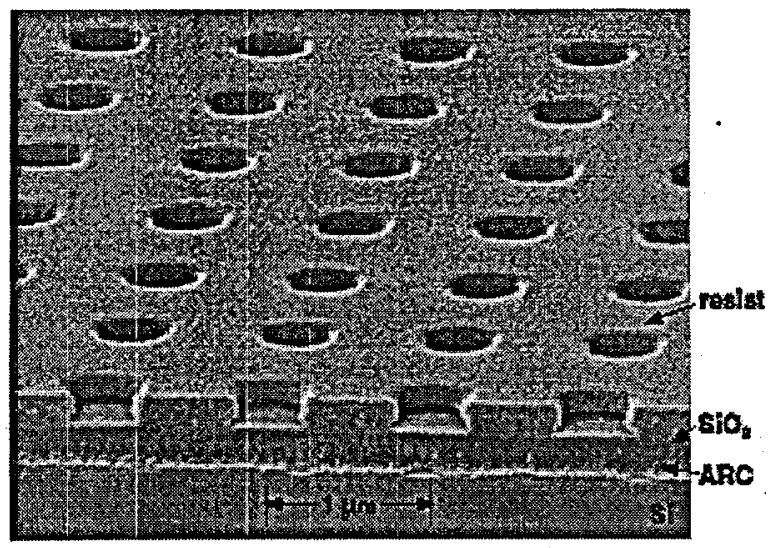

Figure 7 SEM picture of fine pattern etched in to platinum on aluminum oxide.

Sample platinum on sapphire wafers were exposed to high temperature section 6.6) to demonstrate utility at elevated operating temperatures. However, we were not able in phase 1 to obtain emission spectra of these wafers at very high temperatures. 


\section{MEASURE EMITTER OPTICAL PROPERTIES AT LOW TEMPERATURE}

Optical emission spectra of samples shown in figure 6 , heated to $350^{\circ} \mathrm{C}$, were measured by an FTIR spectrometer. Results are shown in figure 8. Calibrated emissivity is compared to the blackbody emission curve at the same temperature. Some important conclusions from this first test are:

- peak wavelength varies proportionately with pattern spacing

- peak height is less than or equal to the black body curve

- emission at wavelengths far removed from the peak is comparable to the polished metal surface (very low)
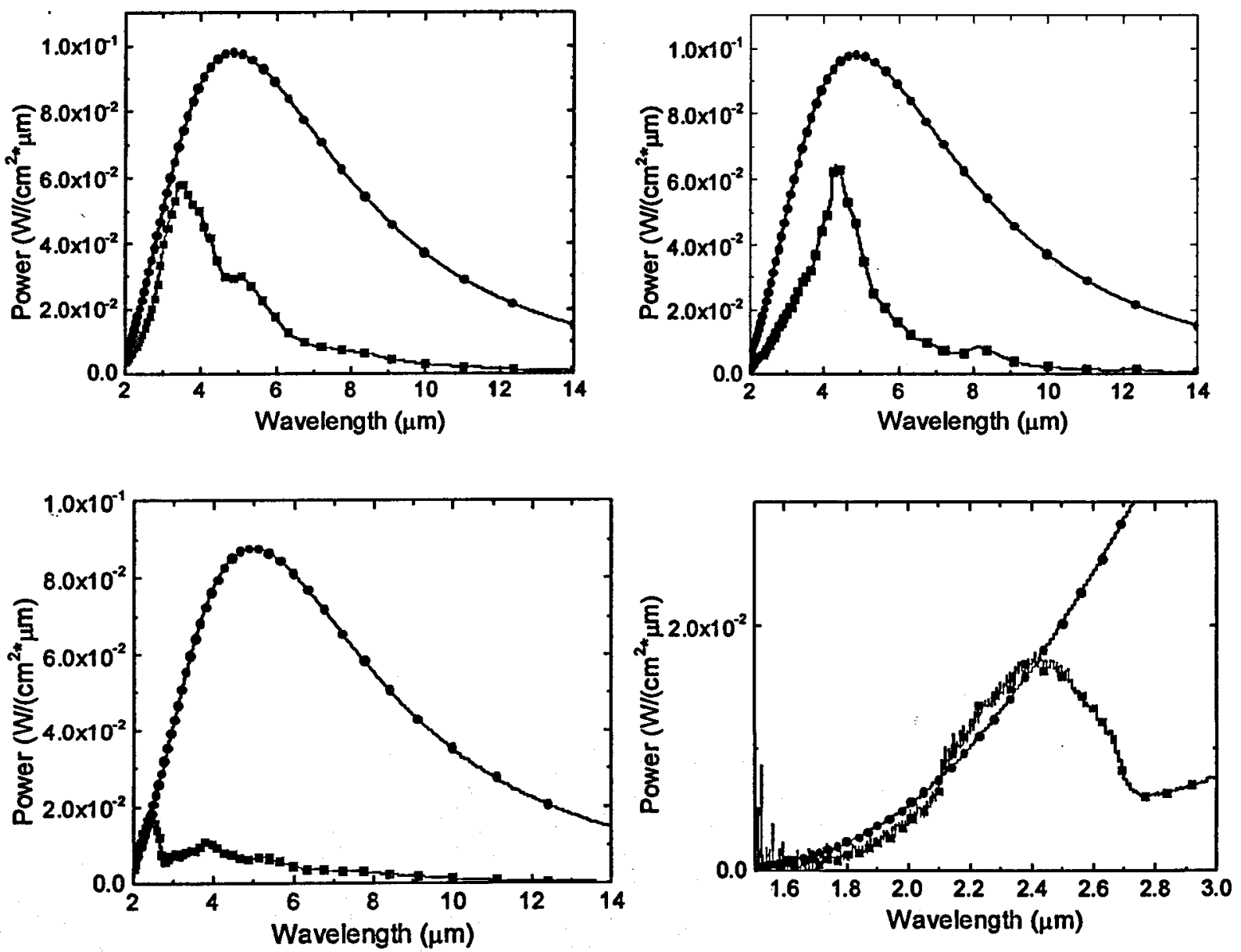

Figure 8 Measured emissivity (lower curves) compared to blackbody at identical temperature. Top left, $2 \mu \mathrm{m}$ squares, $3 \mu \mathrm{m}$ apart. Top right, $3 \mu \mathrm{m}$ square $6 \mu \mathrm{m}$ apart. Bottom left, 1 um round holes 1.5 um apart. Bottom right, expanded view of 1 rom hole 1.5 um apart.

Further experiments examined the variation in emission with angle of viewing. Data in figure 9 were recorded as a function of angle from the surface of the substrate so that normal incidence would $90^{\circ}$. The curve shape is broadened, and the peak height is reduced. Reduction of peak height is due, in part, to a reduction of viewing area from an angle. For example, the area at $45^{\circ}$ would $1 / \mathrm{SQRT}(2)$ or 0.707 that of the maximum at normal incidence. 


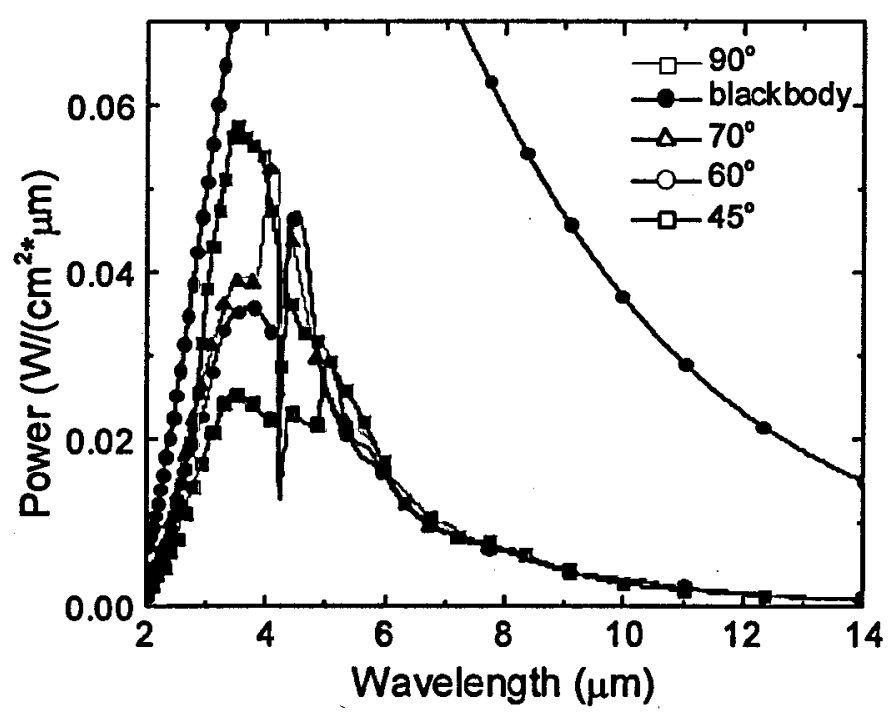

Figure 9 Emission as a function of roll-off angle. Clearly the spectrum is broadened as signal is collected from a greater solid angle.

Data indicate that there is a change in peak wavelength with angle from substrate normal, and angle to the substrate geometry. The latter effect might be lessened with a change in geometry from a square tiling pattern to a hexagonal or Archemedian design.

Cumulative measurements of absorption and emission data as functions of wavelength were related to the geometry of the surface pattern. Figure 10 shows that measured absorption peaks correspond almost exactly to measured emission peaks. The line slope is near 1.0 with a slight offset. This is expected from classical theory. Figure 11 shows that the emission peaks are clearly, linearly related to the surface geometry. We can control the wavelength. This data was used to design the geometry for samples shown in figure 7.

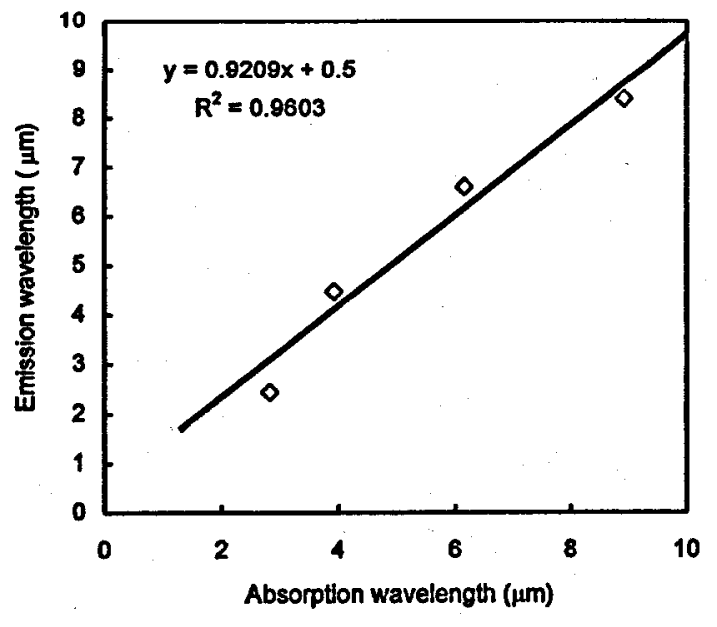

Figure 10 Plot of emission peak wavelength versus absorption peak wavelength for samples shown in figure 12.

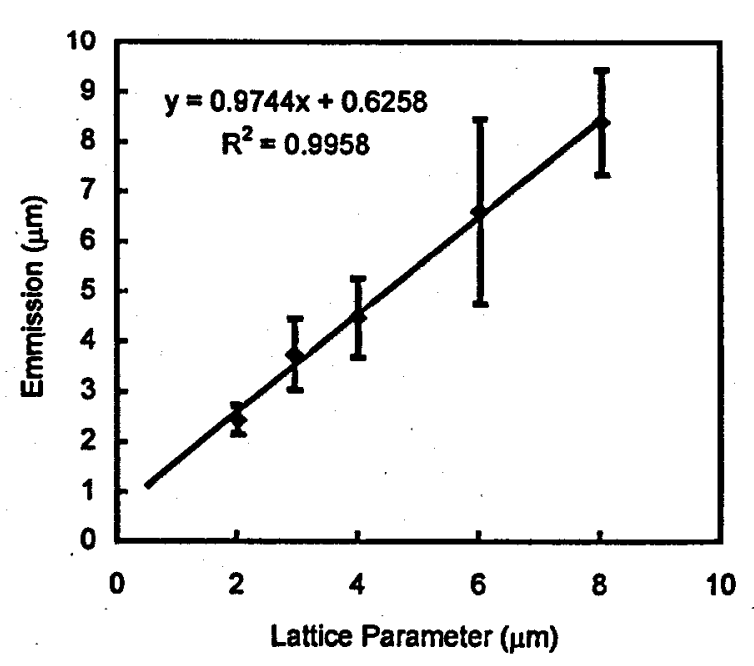

Figure 11 Plot of emission wavelength versus geometrical lattice spacing (center-to-canter) for samples shown in figure 12. 


\section{MEASURE EMITTER OPTICAL PROPERTIES AT HIGH TEMPERATURE}

The last and perhaps most exciting result is the emission at higher temperatures for the $a=3 \mu \mathrm{m}$ sample. Fewer data samples were collected for this experiment, 8 versus 96 , in order to extend the lifetime of the sample. This sample did not have a diffusion barrier between the metal and the silicon, leading to material interactions and a change in optical properties (figure 11). To prevent this interaction, the sample was only briefly brought up in temperature, and immediately scanned.

The results are encouraging, see figure 12. Peak emission wavelength did not change with a change in temperature of the emitter. Power emitted increased according to the black body relationship. The dip at 4.2 microns is absorption by $\mathrm{CO}_{2}$ in the atmosphere. An optimized emitter would function well at $1.4 \mu \mathrm{m}$ peak.

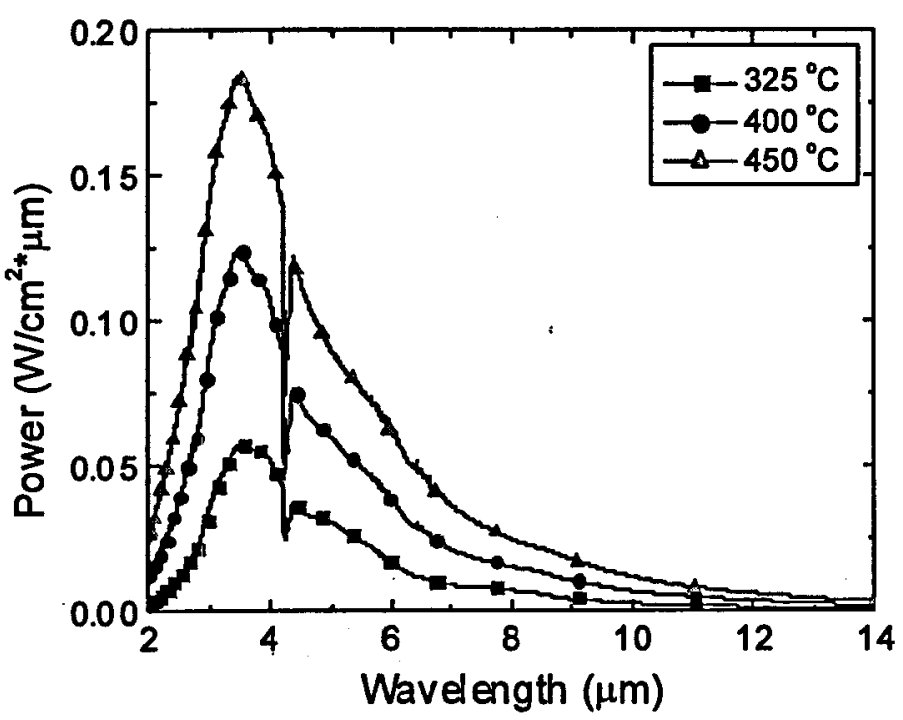

Figure 12 Emission at 325,400 and $450^{\circ} \mathrm{C}$ from phase 1 research. The peak emission does not shift and the entire spectrum increases in intensity.

\section{ASCERTAIN SUITABILITY TO LONG TERM ATMOSPHERIC EXPOSURE AND A LARGE NUMBER OF THERMAL'CYCLES.}

Samples of platinum on sapphire films were furnished to Pittsburg State University for high temperature testing. Certain problems limited the number of hours of testing. Samples with a thin layer titanium film underneath the platinum peeled off. The adhesion layer interacted with platinum and differential thermal expansion of the metallic alloy compared to the substrate created large mechanical stress. By comparison, the annealed pure platinum film, which we originally thought might not stick, survived completely unchanged. There was no detectable difference in optical properties before and after heating for 10 hours at $1,000^{\circ} \mathrm{C}$ in air. 


\section{CONCLUSONS FROM PHASE 1}

- We can fabricate selective emitters using "surface texture' or photonic bandgap structures instead of rare earth oxides.

- Theory matches experiment and indicates that thin conductive films are required for this effect

- The peak emission wavelength can be easily controlled by altering the geometry

- The peak emission wavelength does not change with temperature

- The emission peak shape does not change with temperature

- Structures that would emit radiation below $1.7 \mu \mathrm{m}$ can be fabricated by holographic lithography without having to use electron beams to write a photomask

- Thin platinum films easily survive thermal cycling in air to required temperatures 


\section{References}

1 NIST contract number ATP-99-01-2051, "MEMS-Based Infrared Microsensors".

2 Calculations described at http://cmp/ameslab.gov/ercap/pbg.2001.html

3 T. W. Ebbesen et al, Nature, 391, 667 (1998).

$4 \quad$ H. F. Ghanemi et al, Phys. Rev. B, 58, 6779 (1998).

5 . Tae Jin Kim, et al, Optoics Letters, 24, 256 (1999).

6 Tineke Thio, et al,J. Opt. Soc. Am. B, 16, 1723 (1999).

7 Tinke Thio, et al, Physica B, 279, 90 (2000).

8 D. E. Grupp, et al, Appl. Phys. Lett., 77, 1569 (2000). 